\title{
Noraroe $\mid$ Prevalência de doença renal crônica autorreferida em adultos na Região Metropolitana de Manaus: estudo transversal de base populacional, 2015*
}

doi: 10.5123/S1679-49742020000100003

Prevalence of self-reported chronic kidney disease in adults in the Metropolitan Region of Manaus: a cross-sectional population-based study, 2015

\section{Prevalencia de enfermedad renal crónica autorreferida en adultos en la Región Metropolitana de Manaus: estudio transversal de base poblacional, 2015}

\author{
Ana Wanda Guerra Barreto Marinho' - orcid.org/0000-0002-3291-1584 \\ Taís Freire Galvão² - (1) orcid.org/0000-0003-2072-4834 \\ Marcus Tolentino Silva ${ }^{3}$ - (1) orcid.org/0000-0002-7186-9075 \\ 'Universidade Federal do Amazonas, Faculdade de Medicina, Manaus, AM, Brasil \\ ¿Universidade Estadual de Campinas, Faculdade de Ciências Farmacêuticas, Campinas, SP, Brasil \\ ${ }^{3}$ Universidade de Sorocaba,Programa de Pós-Graduação em Ciências Farmacêuticas, Sorocaba, SP, Brasil
}

\section{Resumo}

Objetivo: estimar a prevalência da doença renal crônica autorreferida e fatores associados em adultos residentes na Região Metropolitana de Manaus, Brasil. Métodos: estudo transversal de base populacional, de 2015, com amostragem probabilística para entrevistar adultos com idade $\geq 18$ anos no domicílio; os fatores associados ao autorrelato de doença renal foram investigados por regressão de Poisson hierarquizada, para calcular as razões de prevalência (RP) e intervalos de confiança de $95 \%\left(\mathrm{IC}_{95 \%}\right)$, considerando-se a amostragem complexa empregada. Resultados: foram entrevistadas 4.001 pessoas - 52,8\% mulheres, 72,2\% pardos, 19,7\% hipertensos; a prevalência de doença renal crônica foi de 2,1\% ( $\mathrm{IC}_{95 \%}$ 1,6;2,5\%), positivamente associada a idade (em anos: 35-44, RP=2,31, $\mathrm{IC}_{95 \%} 1,02 ; 5,21 ; 45-59, \mathrm{RP}=2,52, \mathrm{IC}_{95 \%} 1,10 ; 5,75$; $\left.\geq 60, \mathrm{RP}=2,95, \mathrm{IC}_{95 \%} 1,21 ; 7,16\right)$ e acidente vascular encefálico $\left(\mathrm{RP}=2,20, \mathrm{IC}_{95 \%}\right.$ 1,09;4,45). Conclusão: dois em cada 100 adultos relataram doença renal crônica, mais frequente em mais velhos e com acidente vascular encefálico.

Palavras-chave: Insuficiência Renal Crônica; Adulto; Autorrelato; Prevalência; População; Estudos Transversais.

*Artigo derivado da dissertação de mestrado intitulada'Prevalência de doença renal autorreferida em adultos na Região Metropolitana de Manaus', defendida por Ana Wanda Guerra Barreto Marinho junto ao Programa de Pós-Graduação em Ciências Farmacêuticas da Universidade Federal do Amazonas, Manaus, AM, em 2017. Estudo financiado com recursos do Conselho Nacional de Desenvolvimento Científico e Tecnológico (CNPq)/Ministério da Ciência, Tecnologia, Inovações e Comunicações (MCTIC): Processos n 404990/2013-4 e n ${ }^{\circ} 448093 / 2014-6$. 


\section{Introdução}

A doença renal crônica apresenta distribuição mundial, com prevalência estimada em até 15\% da população, principalmente em países de baixa e média renda. ${ }^{1,2} \mathrm{O}$ diagnóstico precoce da doença é fundamental para que estratégias terapêuticas sejam efetivas para (i) a prevenção ou retardamento de sua progressão e (ii) 0 ingresso do paciente na terapia renal substitutiva - diálise ou transplante renal. ${ }^{3}$ Uma revisão sistemática de estudos brasileiros, publicados até 2017, estimou que $3 \mathrm{em}$ cada 100 brasileiros seriam portadores da doença e 5 em cada 10 mil se submeteriam a alguma modalidade dialítica. ${ }^{4}$

As principais causas de doença renal crônica são a hipertensão arterial e o diabetes mellitus, que predispõem a complicações vasculares, como infarto agudo do miocárdio e acidente vascular encefálico. ${ }^{5}$ Pessoas com nefropatias apresentam maiores riscos de mortalidade por doenças cardiovasculares, em todos os estágios evolutivos. ${ }^{6}$

\section{As principais causas de doença renal crônica são a hipertensão arterial e o diabetes mellitus, que predispõem a complicações vasculares, como infarto agudo do miocárdio e acidente vascular encefálico}

A diálise - tratamento do último estágio evolutivo da doença renal crônica - é um procedimento de alto custo. No Brasil, essa terapia é quase exclusivamente oferecida pelo Sistema Único de Saúde (SUS), de forma direta ou indireta. ${ }^{7}$ Conhecer a prevalência da doença renal crônica é importante para o planejamento de ações de prevenção secundária e promoção da saúde entre a população. ${ }^{1}$ Para localidades de menor densidade de profissionais e serviços de saúde, como no estado do Amazonas, essa investigação adquire particular relevância.

0 objetivo da presente pesquisa foi estimar a prevalência da doença renal crônica e fatores associados, em adultos residentes na Região Metropolitana de Manaus, Amazonas, Brasil.

\section{Métodos}

Trata-se de estudo transversal de base populacional, realizado nos meses de maio a julho de 2015 , com adultos residentes na Região Metropolitana de Manaus, constituída de oito municípios - Careiro da Várzea, Iranduba, Itacoatiara, Manacapuru, Novo Airão, Presidente Figueiredo, Rio Preto da Eva e Manaus - e população estimada em 2,1 milhões de residentes, mais de $60 \%$ dos habitantes do estado do Amazonas, de acordo com o Censo Demográfico 2010. ${ }^{8}$ Em 2013, a Região Metropolitana de Manaus apresentou índice de desenvolvimento humano (IDH) de $0,720{ }^{9}$

A presente análise faz parte de pesquisa com 0 propósito de estimar o uso de insumos e serviços de saúde na região. ${ }^{10}$

Adultos com idade $\geq 18$ anos foram selecionados por meio de amostragem probabilística, em três estágios. ${ }^{8}$ No primeiro estágio, foram sorteados 400 setores primários e 20 de reposição, entre os 2.647 setores censitários urbanos da região metropolitana de Manaus. ${ }^{8}$ No segundo estágio, empregou-se amostragem sistemática para selecionar os domicílios: um número entre 1 e 20 foi sorteado para determinar o primeiro domicílio, a partir do qual, a cada 20 domicílios, um foi visitado até se completarem dez entrevistas por setor. Todos os moradores elegíveis presentes foram cadastrados no dispositivo eletrônico da entrevista e um foi sorteado a partir de cotas predefinidas de sexo e idade, baseadas nas estimativas oficiais. ${ }^{10}$

0 tamanho da amostra foi calculado em 4.001 adultos, partindo de estimativa conservadora de 50\% no uso de serviço de saúde, nível de confiança de $95 \%$, precisão absoluta de $2 \%$, efeito do desenho de 1,5 e 2.106.322 adultos na região. ${ }^{8}$

Entrevistadores treinados coletaram os dados utilizando-se de questionários semiestruturados, propostos aos participantes em entrevistas face a face. Todas as variáveis foram autorreferidas. 0 desfecho primário foi a prevalência autorreferida de doença renal crônica, aferida por meio da seguinte pergunta:

"Algum médico já lhe deu o diagnóstico de doença renal crônica?" (sim; não).

As demais variáveis adotadas nesta análise foram:

a) Sociodemográficas

- sexo (masculino; feminino);

- idade (em anos); 
- peso (em kg);

- altura (em cm);

- nível educacional (superior ou mais; médio;

fundamental; menos que fundamental);

- raça/cor da pele (branca; preta; amarela; parda; indígena);

- classificação econômica, de acordo com 0 Critério Brasil de classificação econômica (A, B, C ou D/E; onde A é o extrato mais rico e D/E 0 mais pobre);

- situação de trabalho (formal; informal; aposentado; estudante/dona de casa; não trabalha);

- localização da cidade de residência no estado (interior; capital);

b) Clínicas

- doenças crônicas autorreferidas - hipertensão, diabetes, cardiopatia, hipercolesterolemia e acidente vascular encefálico (sim; não);

- estado de saúde (muito bom; bom; regular; ruim; muito ruim);

- índice de massa corporal (IMC, em quilogramas por metro quadrado $\left.\left[\mathrm{kg} / \mathrm{m}^{2}\right]:<25 ; 25-29,9 ; \geq 30\right)$. Os dados foram descritos em frequências absolutas e relativas. Razões de prevalência (RP) de doença renal segundo categorias das variáveis do estudo e intervalos de confiança de $95 \%\left(\mathrm{IC}_{95 \%}\right.$ ) foram calculados em análise bivariável. Utilizou-se regressão de Poisson com variância robusta para calcular as RPs ajustadas, seguindo modelo hierárquico considerando as variáveis distais e proximais ao desfecho. ${ }^{11}$ 0 primeiro bloco de questões foi composto pelas variáveis sociais (classificação econômica; situação de trabalho; escolaridade; localização da cidade). No segundo bloco, foram adicionadas as variáveis demográficas (sexo; idade; raça/cor da pele). As variáveis clínicas (doenças crônicas; estado de saúde; IMC) foram adicionadas no terceiro bloco de análise. Cada bloco foi ajustado pelas variáveis do bloco e do nível anterior. A significância estatística das variáveis com mais de duas categorias foi calculada pelo teste de Wald, após análise ajustada de cada bloco. As análises foram realizadas utilizande-se o software Stata 14.2 (StataCorp, College Station, Texas, USA), sendo considerado o delineamento complexo da amostra (comando svy).

0 projeto da pesquisa foi aprovado pelo Comitê de Ética em Pesquisa da Universidade Federal do Amazo- nas (CEP/UFAN): Processo $n^{0}$ 974.428, de 3 de março de 2015 (Certificado de Apresentação para Apreciação Ética [CAAE] n ${ }^{\circ}$ 42203615.4.0000.5020). 0 Termo de Consentimento Livre e Esclarecido foi assinado por todos os participantes, como condição para realização da entrevista.

\section{Resultados}

Foram incluídos na pesquisa 4.001 adultos (Tabela 1). A prevalência autorreferida de doença renal foi de 2,1\% ( $\left.\mathrm{IC}_{95 \%}, 1,6 ; 2,5\right)$. Na amostra, houve discreto predomínio de mulheres $(52,8 \%)$, adultos jovens, de 25 a 34 anos (28,8\%), pardos (72,1\%; apenas $1,0 \%$ se consideravam indígenas), indivíduos da classe social C (35,6\%), com ensino médio (47,5\%) e na situação de trabalho informal $(28,8 \%)$. A maioria dos entrevistados residia em Manaus (86,9\%). As doenças crônicas mais referidas foram hipertensão $(19,7 \%)$ e diabetes mellitus (6,2\%). Mais da metade dos entrevistados referiram bom estado de saúde $(54,3 \%)$.

A análise bruta revelou que a doença renal crônica foi significativamente mais frequente entre pessoas mais velhas, aposentadas e autodeclaradas de raça/ cor da pele parda (Tabela 2). Fatores clínicos positivamente associados incluíram hipertensão, diabetes, cardiopatia, hipercolesterolemia e acidente vascular encefálico $(\mathrm{p}<0,001)$. Após ajustes, a doença renal crônica foi positivamente associada a idade (em anos: 35-44, RP=2,31, IC $\mathrm{C}_{95 \%} 1,02 ; 5,21 ; 45-59, \mathrm{RP}=2,52$, $\left.\mathrm{IC}_{95 \%} 1,10 ; 5,75 ; \mathrm{e} \geq 60, \mathrm{RP}=2,95, \mathrm{IC}_{95 \%} 1,21 ; 7,16\right)$, ser aposentado $\left(\mathrm{RP}=2,18, \mathrm{IC}_{95 \%}, 1,05 ; 4,51\right)$ e ter sofrido acidente vascular encefálico $\left(\mathrm{RP}=2,20, \mathrm{IC}_{95 \%}\right.$ 1,09;4,45). Na comparação com nível de educação superior, pessoas com nível médio tiveram - significativamente - menor prevalência de doença renal autorreferida $\left(\mathrm{RP}=0,34, \mathrm{IC}_{95 \%} 0,11 ; 0,99\right)$.

\section{Discussão}

Dois adultos em cada 100 residentes da Região Metropolitana de Manaus referiram ser portadores de doença renal crônica, correspondendo a mais de 40.000 pessoas. A enfermidade foi positivamente associada a maior idade, aposentadoria e acidente vascular encefálico. A prevalência encontrada foi um pouco superior à observada para o conjunto do país, tendo-se como referência os dados da Pesquisa Nacional de 
Tabela 1 - Características da população incluída ( $n=4.001$ ) e prevalência de doença renal crônica autorreferida na população da Região Metropolitana de Manaus, Amazonas, 2015

\begin{tabular}{|c|c|c|c|}
\hline Variável & $\mathbf{n}$ & $\%^{\mathrm{a}}$ & $\begin{array}{c}\text { Prevalência } \\
\%\left({ }^{\left(I C_{95 \%}\right.}{ }^{b}\right)\end{array}$ \\
\hline \multicolumn{4}{|l|}{ Sexo } \\
\hline Masculino & 1.888 & 47,2 & $1,7(1,2 ; 2,4)$ \\
\hline Feminino & 2.113 & 52,8 & $2,4(1,8 ; 3,1)$ \\
\hline \multicolumn{4}{|l|}{ Faixa etária (em anos) } \\
\hline $18-24$ & 838 & 20,9 & $1,0(0,5 ; 1,9)$ \\
\hline $25-34$ & 1.152 & 28,8 & $0,7(0,3 ; 1,4)$ \\
\hline $35-44$ & 843 & 21,1 & $2,3(1,5 ; 3,6)$ \\
\hline $45-59$ & 772 & 19,3 & $2,9(2,0 ; 4,4)$ \\
\hline$\geq 60$ & 396 & 9,9 & $6,0(4,0 ; 8,8)$ \\
\hline \multicolumn{4}{|l|}{ Raça/cor da pele } \\
\hline Branco & 636 & 15,9 & $1,1(0,5 ; 2,2)$ \\
\hline Preto & 300 & 7,5 & $0,7(0,2 ; 2,6)$ \\
\hline Amarelo & 138 & 3,5 & $2,9(1,1 ; 7,4)$ \\
\hline Pardo & 2.886 & 72,1 & $2,4(1,9 ; 3,0)$ \\
\hline Indígena & 41 & 1,0 & $2,4(0,3 ; 15,4)$ \\
\hline \multicolumn{4}{|l|}{ Classificação econômica } \\
\hline$A$ & 629 & 15,7 & $0,9(0,4 ; 2,1)$ \\
\hline B & 862 & 21,5 & $1,0(0,5 ; 2,0)$ \\
\hline C & 1.423 & 35,6 & $2,2(1,5 ; 3,1)$ \\
\hline $\mathrm{D} / \mathrm{E}$ & 1.087 & 27,2 & $3,4(2,4 ; 4,6)$ \\
\hline \multicolumn{4}{|l|}{ Educação } \\
\hline Superior ou mais & 158 & 4,0 & $2,5(0,9 ; 6,4)$ \\
\hline Médio & 1.903 & 47,5 & $1,0(0,6 ; 1,6)$ \\
\hline Fundamental & 649 & 16,2 & $1,4(0,7 ; 2,6)$ \\
\hline Menos que fundamental & 1.291 & 32,3 & $3,9(3,0 ; 5,1)$ \\
\hline \multicolumn{4}{|l|}{ Situação de trabalho } \\
\hline Formal & 761 & 19,0 & $1,6(0,9 ; 2,7)$ \\
\hline Informal & 1.149 & 28,8 & $1,5(1,0 ; 2,4)$ \\
\hline Aposentado & 315 & 7,9 & $6,3(4,1 ; 9,6)$ \\
\hline Estudante/dona de casa & 1.199 & 29,9 & $2,3(1,6 ; 3,3)$ \\
\hline Não trabalha & 577 & 14,4 & $1,0(0,5 ; 2,3)$ \\
\hline \multicolumn{4}{|l|}{ Cidade de residência } \\
\hline Interior & 522 & 13,1 & $1,4(0,7 ; 2,8)$ \\
\hline Capital & 3.479 & 86,9 & $2,2(1,7 ; 2,7)$ \\
\hline
\end{tabular}

a) Percentual ponderado pela amostragem complexa empregada. b) IC $\mathrm{I}_{95 \%}$ intervalo de confiança de $95 \%$. 
Tabela 1 - Características da população incluída $(n=4.001)$ e prevalência de doença renal crônica autorreferida na população da Região Metropolitana de Manaus, Amazonas, 2015

\begin{tabular}{|c|c|c|c|}
\hline Variável & $\mathrm{n}$ & $\%^{\mathrm{a}}$ & $\begin{array}{c}\text { Prevalência } \\
\%\left(\text { IC }_{95 \%}{ }^{\circ}\right)\end{array}$ \\
\hline \multicolumn{4}{|l|}{ Doenças crônicas } \\
\hline Hipertensão & 787 & 19,7 & $4,1(2,9 ; 5,7)$ \\
\hline Diabetes & 245 & 6,2 & $5,7(3,4 ; 9,4)$ \\
\hline Cardiopatia & 203 & 5,1 & $6,9(4,1 ; 11,3)$ \\
\hline Hipercolesterolemia & 596 & 14,9 & $5,0(3,5 ; 7,1)$ \\
\hline Acidente vascular encefálico & 104 & 2,6 & $10,5(5,9 ; 18,0)$ \\
\hline \multicolumn{4}{|l|}{ Estado de saúde } \\
\hline Muito bom & 471 & 11,8 & $0,8(0,3 ; 2,2)$ \\
\hline Bom & 2.175 & 54,3 & $1,4(0,9 ; 1,9)$ \\
\hline Regular & 1.108 & 27,6 & $2,9(2,0 ; 4,1)$ \\
\hline Ruim & 193 & 4,9 & $6,2(3,5 ; 10,5)$ \\
\hline Muito ruim & 54 & 1,4 & $9,3(3,9 ; 20,4)$ \\
\hline \multicolumn{4}{|c|}{ Índice de massa corpórea $\left(\mathrm{kg} / \mathrm{m}^{2}\right)$} \\
\hline$<25$ & 1.591 & 39,8 & $2,0(1,4 ; 2,8)$ \\
\hline $25-29,99$ & 1.554 & 38,9 & $1,6(1,1 ; 2,3)$ \\
\hline$\geq 30$ & 852 & 21,3 & $3,0(2,1 ; 4,4)$ \\
\hline
\end{tabular}

a) Percentual ponderado pela amostragem complexa empregada.

b) $\mathrm{IC}_{95 \%}$ : intervalo de confiança de $95 \%$.

Saúde (PNS) de 2013. A PNS, a exemplo do presente estudo, tampouco observou diferenças quanto a sexo, escolaridade e raça/cor da pele. ${ }^{11}$

O desfecho primário desta análise baseou-se no autorrelato de uma doença de características silenciosas, passível de ser confundida com doenças urológicas, levando a erro de classificação no desfecho. 0 emprego de ferramentas diagnósticas, baseadas em análise laboratorial com a dosagem de creatinina sérica e pesquisa de proteinúria para comprovação da doença renal, aumentaria a confiança nos resultados. ${ }^{12}$

A prevalência de doença renal crônica cresceu com 0 avanço da idade e também se mostrou mais frequente entre aposentados. Estes resultados podem refletir tanto o processo natural de envelhecimento e senescência renal como os danos promovidos pelas comorbidades adquiridas ao longo da vida, a exemplo do diabetes mellitus e da hipertensão arterial. ${ }^{13}$

De acordo com este estudo, somente 0 acidente vascular encefálico revelou-se associado à doença renal. Tal condição resulta, principalmente, de hipertensão arterial não controlada e outros problemas cardiovasculares, igualmente fatores de risco para doença renal crônica. ${ }^{5}$ Doenças cardiovasculares em pacientes com doença renal crônica são mais frequentes e mais graves que na população sem comprometimento renal; certamente, 0 acidente vascular encefálico contribui para o excesso de risco de mortalidade observado. ${ }^{1}$ Possivelmente devido à baixa prevalência de doença renal crônica, não se observou associação entre a doença e a maioria das variáveis estudadas. A hipertensão foi a doença crônica mais autorreferida pela população estudada, e ainda assim não se mostrou associada à doença renal.

A associação entre doença renal crônica e classe econômica não foi significativa. Contudo, é sabido que pessoas pertencentes à classe social inferior, em sociedades desiguais, estão mais expostas aos desfechos desfavoráveis das doenças crônicas, revelando o caráter social da doença. ${ }^{14} \mathrm{O}$ diagnóstico e 0 tratamento adequado da doença dependem do acesso aos serviços de saúde, das 
Tabela 2 - Razão de prevalência e intervalo de confiança de 95\% para doença renal crônica autorreferida na população ( $n=4.001$ ) da Região Metropolitana de Manaus, Amazonas, 2015

\begin{tabular}{|c|c|c|c|c|c|}
\hline Variável & $\begin{array}{l}\text { RPa bruta } \\
\left(\mathrm{IC}_{95 \%}^{\mathrm{b}}\right)\end{array}$ & $\begin{array}{l}\text { Valor } \\
\text { pc }\end{array}$ & $\begin{array}{l}\text { RPa ajustada } \\
\left(\mathrm{IC}_{95 \%}^{\mathrm{b}}\right)\end{array}$ & $\begin{array}{l}\text { Valor } \\
p^{c}\end{array}$ & Bloco $^{d}$ \\
\hline Sexo & & 0,112 & & 0,668 & $2^{0}$ \\
\hline Masculino & 1,00 & & 1,00 & & \\
\hline Feminino & $1,43(0,92 ; 2,21)$ & & $1,05(0,64 ; 1,57)$ & & \\
\hline Faixa etária (em anos) & & $<0,001$ & & 0,004 & $2^{0}$ \\
\hline $18-24$ & 1,00 & & 1,00 & & \\
\hline $25-34$ & $0,72(0,27 ; 1,92)$ & & $0,72(0,27 ; 1,95)$ & & \\
\hline $35-44$ & $2,46(1,09 ; 5,55)$ & & $2,31(1,02 ; 5,21)$ & & \\
\hline $45-59$ & $3,08(1,39 ; 6,85)$ & & $2,52(1,10 ; 5,75)$ & & \\
\hline$\geq 60$ & $6,24(2,83 ; 13,78)$ & & $2,95(1,21 ; 7,16)$ & & \\
\hline Raça/cor da pele & & 0,125 & & 0,231 & $2^{0}$ \\
\hline Branco & 1,00 & & 1,00 & & \\
\hline Preto & $0,61(0,13 ; 2,92)$ & & $0,51(0,10 ; 2,44)$ & & \\
\hline Amarelo & $2,68(0,79 ; 9,04)$ & & $2,34(0,70 ; 7,76)$ & & \\
\hline Pardo & $2,20(1,02 ; 4,77)$ & & $1,77(0,81 ; 3,91)$ & & \\
\hline Indígena & $2,27(0,29 ; 18,01)$ & & $1,39(0,20 ; 9,57)$ & & \\
\hline Classificação econômica & & 0,001 & & 0,135 & $1^{0}$ \\
\hline A & 1,00 & & 1,00 & & \\
\hline B & $1,11(0,40 ; 3,11)$ & & $1,15(0,42 ; 3,17)$ & & \\
\hline C & $2,30(0,97 ; 5,50)$ & & $2,11(0,88 ; 5,08)$ & & \\
\hline $\mathrm{D} / \mathrm{E}$ & $3,59(1,52 ; 8,46)$ & & $2,29(0,95 ; 5,55)$ & & \\
\hline Educação & & $<0,001$ & & 0,006 & $1^{0}$ \\
\hline Superior ou mais & 1,00 & & 1,00 & & \\
\hline Médio & $0,41(0,14 ; 1,18)$ & & $0,34(0,11 ; 0,99)$ & & \\
\hline Fundamental & $0,56(0,18 ; 1,81)$ & & $0,41(0,13 ; 1,27)$ & & \\
\hline Menos que fundamental & $1,59(0,58 ; 4,35)$ & & $0,90(0,32 ; 2,57)$ & & \\
\hline Situação de trabalho & & $<0,001$ & & 0,276 & $1^{0}$ \\
\hline Formal & 1,00 & & 1,00 & & \\
\hline Informal & $0,98(0,48 ; 2,03)$ & & $0,75(0,37 ; 1,52)$ & & \\
\hline Aposentado & $4,04(2,00 ; 8,17)$ & & $2,18(1,05 ; 4,51)$ & & \\
\hline Estudante/dona de casa & $1,45(0,74 ; 2,84)$ & & $1,08(0,55 ; 2,13)$ & & \\
\hline Não trabalha & $0,66(0,25 ; 1,76)$ & & $0,56(0,21 ; 1,46)$ & & \\
\hline Cidade de residência & & 0,244 & & 0,138 & $1^{0}$ \\
\hline Interior & 1,00 & & 1,00 & & \\
\hline Capital & $1,54(0,74 ; 3,18)$ & & $1,72(0,84 ; 3,50)$ & & \\
\hline $\begin{array}{l}\text { a) RP: rrazão de prevalência. } \\
\text { b) IC } \\
\text { cosstintervalo de confiança de } 95 \% \\
\text { c) Teste de Wald. } \\
\text { d) Bloco de entrada na análise, ajustaa }\end{array}$ & & & & & continua \\
\hline
\end{tabular}


continuação

Tabela 2 - Razão de prevalência e intervalo de confiança de 95\% para doença renal crônica autorreferida na população ( $n=4.001$ ) da Região Metropolitana de Manaus, Amazonas, 2015

\begin{tabular}{|c|c|c|c|c|c|}
\hline Variável & $\begin{array}{c}\text { RPa bruta } \\
\left(\mathrm{IC}_{95 \%}{ }^{\mathrm{b}}\right)\end{array}$ & $\begin{array}{c}\text { Valor } \\
p^{c}\end{array}$ & $\begin{array}{c}\text { RPa ajustada } \\
\quad\left(\mathrm{IC}_{95 \%}{ }^{b}\right)\end{array}$ & $\begin{array}{c}\text { Valor } \\
p^{c}\end{array}$ & Bloco $^{d}$ \\
\hline \multicolumn{6}{|l|}{ Doenças crônicas ${ }^{e}$} \\
\hline Hipertensão & $2,59(1,68 ; 4,01)$ & $<0,001$ & $0,93(0,53 ; 1,65)$ & 0,808 & 30 \\
\hline Diabetes & $3,12(1,78 ; 5,46)$ & $<0,001$ & $1,05(0,59 ; 1,87)$ & 0,860 & 30 \\
\hline Cardiopatia & $3,84(2,20 ; 6,70)$ & $<0,001$ & $1,46(0,76 ; 2,83)$ & 0,255 & $3^{\circ}$ \\
\hline Hipercolesterolemia & $3,28(2,11 ; 5,09)$ & $<0,001$ & $1,59(0,91 ; 2,76)$ & 0,103 & $3^{0}$ \\
\hline Acidente vascular encefálico & $5,75(3,14 ; 10,53)$ & $<0,001$ & $2,20(1,09 ; 4,45)$ & 0,029 & $3^{0}$ \\
\hline Estado de saúde & & $<0,001$ & & 0,175 & 30 \\
\hline Muito bom & 1,00 & & 1,00 & & \\
\hline Bom & $1,61(0,57 ; 4,54)$ & & $1,07(0,37 ; 3,11)$ & & \\
\hline Regular & $3,42(1,22 ; 9,63)$ & & $1,41(0,47 ; 4,27)$ & & \\
\hline Ruim & $7,31(2,39 ; 22,41)$ & & $2,23(0,66 ; 7,51)$ & & \\
\hline Muito ruim & $10,99(3,04 ; 39,72)$ & & $2,71(0,70 ; 10,49)$ & & \\
\hline Índice de massa corpórea (kg/m²) & & 0,059 & & 0,344 & $3^{\circ}$ \\
\hline$<25$ & 1,00 & & 1,00 & & \\
\hline $25-29,9$ & $0,79(0,47 ; 1,33)$ & & $0,76(0,45 ; 1,27)$ & & \\
\hline$\geq 30$ & $1,51(0,91 ; 2,52)$ & & $1,12(0,66 ; 1,90)$ & & \\
\hline
\end{tabular}

a) RP: razão de prevalência.

b) IC : intervalo de confiança de $95 \%$

c) Teste de Wald.

d) Bloco de entrada na análise, ajustado pelas variáveis do bloco e do nível acima.

e) Referência:ausência da doença.

políticas públicas para controle do diabetes mellitus e da hipertensão, e de educação básica em saúde.

Uma Atenção Básica organizada é essencial para a prevenção e controle precoce da doença renal crônica. ${ }^{12}$ As principais causas da doença são hipertensão e diabetes, os quais, quando bem controlados na Atenção Básica, inibem o surgimento da doença renal e retardam 0 início da diálise, ${ }^{15,16}$ além de tal controle contribuir para a redução das complicações cardiovasculares, como infarto e acidente vascular encefálico, que podem levar a óbito. ${ }^{15}$

A baixa prevalência da doença renal, encontrada neste estudo, pode refletir o desconhecimento do próprio estado de saúde, dada a dificuldade de acesso ao sistema de saúde, além de uma demanda não atendida de exames laboratoriais, como dosagem de creatinina sérica e proteinúria para se confirmar a injúria renal. ${ }^{12,17}$

Manaus é a única cidade do estado do Amazonas a oferecer terapia renal substitutiva para pessoas com doença renal em estágio avançado. ${ }^{7}$ Estimativa da Sociedade Brasileira de Nefrologia sugere que a prevalência de pacientes submetidos a diálise no Amazonas é de 229 por cada milhão de habitantes. ${ }^{7}$ Provavelmente, os dados estão subestimados, haja vista o isolamento geográfico característico das populações distribuídas pela extensa região Norte e a dificuldade de acesso aos serviços de saúde. ${ }^{10} 0$ s residentes na região metropolitana estuda$\mathrm{da}$, entretanto, seriam justamente aqueles com mais facilidade de acesso a serviços de saúde e tratamento dialítico no estado.

Em conclusão, a doença renal crônica foi autorreferida por 2 em cada 100 adultos da região metropolitana de Manaus. A relativamente baixa consciência da doença pode refletir pouco acesso aos serviços de saúde, em especial à Atenção Básica. Estudos representativos, com emprego de ferramentas diagnósticas, são necessários para melhor estimar a prevalência da doença renal crônica na região. 


\section{Contribuição dos autores}

Silva MT e Galvão TF delinearam a pesquisa. Marinho AWGB, Silva MT e Galvão TF analisaram os dados. Marinho AWGB e Galvão TF interpretaram os dados e

\section{Referências}

1. Levin A, Tonelli M, Bonventre J, Coresh J, Donner JA, Fogo AB, et al. Global kidney health 2017 and beyond: a roadmap for closing gaps in care, research, and policy. Lancet [Internet]. 2017 Oct [cited 2020 Jan 10];390(10105):1888-917. Available from: https:// www.thelancet.com/journals/lancet/article/PIIS01406736(17)30788-2/fulltext. doi: 10.1016/S01406736(17)30788-2

2. Hill NR, Fatoba ST, Oke JL, Hirst JA, O'Callaghan CA, Lasserson DS, et al. Global prevalence of chronic kidney disease-a systematic review and metaanalysis. PloS One [Internet]. 2016 Jul [cited 2020 Jan 10];11(7):e0158765. Available from: https:// journals.plos.org/plosone/article?id=10.1371/journal. pone.0158765. doi: 10.1371/journal.pone. 0158765

3. Ferraz FHRP, Rodrigues CIS, Gatto GC, Sá NMd. Differences and inequalities in relation to access to renal replacement therapy in the BRICS countries. Ciênc Saúde Colet [Internet]. 2017 Jul [cited 2020 Jan 10];22(7):2175-85. Available from: http://www.scielo. br/pdf/csc/v22n7/en_1413-8123-csc-22-07-2175.pdf. doi: 10.1590/1413-81232017227.00662017

4. Marinho AWGB, Penha AP, Silva MT, Galvão TF. Prevalência de doença renal crônica em adultos no Brasil: revisão sistemática da literatura. Cad Saúde Colet [Internet]. 2017 jul [citado 2020 jan 10];25(3):37988. Disponível em: http://www.scielo.br/pdf/cadsc/ v25n3/1414-462X-cadsc-1414-462X201700030134. pdf. doi: 10.1590/1414-462x201700030134

5. Gansevoort RT, Correa-Rotter R, Hemmelgarn BR, Jafar TH, Heerspink HJL, Mann JF, et al. Chronic kidney disease and cardiovascular risk: epidemiology, mechanisms, and prevention. Lancet [Internet]. 2013 Jul [cited 2020 Jan 10];382(9889):339-52. Available from: https://www.thelancet.com/journals/lancet/ article/PIIS0140-6736(13)60595-4/fulltext. doi: 10.1016/S0140-6736(13)60595-4

6. Hill NR, Fatoba ST, Oke JL, Hirst JA, O'Callaghan CA, Lasserson DS, et al. Global prevalence of chronic kidney disease - a systematic review and meta- redigiram a primeira versão do manuscrito. Marinho AWGB, Silva MT e Galvão TF revisaram criticamente o manuscrito. Todos os autores aprovaram a versão final e são responsáveis por todos os aspectos do trabalho, incluindo a garantia de sua precisão e integridade.

analysis. PLoS One [Internet]. 2016 Jul [cited 2020 Jan 10];11(7):e0158765. Available from: https:// journals.plos.org/plosone/article?id=10.1371/journal. pone.0158765. doi: 10.1371/journal.pone.0158765

7. Sesso RC, Lopes AA, Thomé FS, Lugon JR, Martins CT. Brazilian chronic dialysis survey 2016. J Bras Nefrol [Internet]. 2017 Jul-Set [cited 2020 Jan 10];39(3):261-6. Available from: http://www.scielo.br/ pdf/jbn/v39n3/0101-2800-jbn-39-03-0261.pdf. doi: 10.5935/0101-2800.20170049

8. Instituto Brasileiro de Geografia e Estatística. Censo demográfico de 2010. Amazonas [Internet]. Rio de Janiero: Instituto Brasileiro de Geografia e Estatística; 2010 [citado 2020 jan 10]. Disponível em: http://www.censo2010.ibge.gov.br/sinopse/index. php?uf $=13 \&$ dados $=4$

9. Programa das Nações Unidas para Desenvolvimento. Atlas do Desenvolvimento Humano no Brasil: PNUD [Internet]. [S1]: PNUD; 2013 [citado 2020 jan 10]. Disponivel em: http://atlasbrasil.org.br/2013

10. Silva MT, Galvao TF. Use of health services among adults living in Manaus Metropolitan Region, Brazil: population-based survey, 2015. Epidemiol Serv Saúde [Internet]. 2017 Oct-Dec [cited 2020 Jan 10];26(4):725-34. Available from: http:// www.scielo.br/pdf/ress/v26n4/en_2237-9622ress-s1679-49742017000400005.pdf. doi: 10.5123/ S1679-49742017000400005

11. Moura L, Andrade SSCA, Malta DC, Pereira CA, Passos JEF. Prevalence of self-reported chronic kidney disease in Brazil: National Health Survey of 2013. Rev Bras Epidemiol [Internet]. 2015 Dec [cited 2020 Jan 10];18 Suppl 2:181-91. Availble from: http://www.scielo.br/ pdf/rbepid/v18s2/en_1980-5497-rbepid-18-s2-00181. pdf. doi: 10.1590/1980-5497201500060016

12. Sá HO. Chronic Kidney Disease (CKD) prevention or the urgency of a national policy of screening and early treatment. Port J Nephrol Hypert [Internet]. 2013 Feb [cited 2020 Jan 10];27(1):9-12. 
Available from: https://pdfs.semanticscholar.org/ b9ee/255bbdd1bbd265f3fb85dafe2c2666864a98. pdf?_ga=2.113048452.1470758040.1579091878783148302.1544653156

13. Eckardt KU, Coresh J, Devuyst O, Johnson RJ, Kottgen A, Levey AS, et al. Evolving importance of kidney disease: from subspecialty to global health burden. Lancet [Internet]. 2013 Jul [cited 2020 Jan 10];382(9887):158-69. Available from: https:// www.thelancet.com/journals/lancet/article/PIIS01406736(13)60439-0/fulltext. doi: 10.1016/S01406736(13)60439-0

14. Tirapani LS, Pinheiro HS, Mansur HN, Oliveira Dd, Huaira RMNH, Huaira CC, et al. Impact of social vulnerability on the outcomes of predialysis chronic kidney disease patients in an interdisciplinary center. Braz J Nephrol [Internet]. 2015 Jan-Mar [cited 2020 Jan 10];37(1):19-26. Available from: http://www.scielo. br/pdf/jbn/v37n1/0101-2800-jbn-37-01-0019.pdf. doi: 10.5935/0101-2800.20150004

15. Jha V, Garcia-Garcia G, Iseki K, Li Z, Naicker S, Plattner $\mathrm{B}$, et al. Chronic kidney disease: global dimension and

\section{Abstract}

Objective: to estimate the prevalence of self-reported chronic kidney disease and associated factors in adults living in the Metropolitan Region of Manaus, Brazil. Methods: a cross-sectional population-based study conducted in 2015, with probabilistic sampling to select adults $\geq 18$ years; participants were interviewed at home; factors associated with self-reported chronic kidney disease were investigated by Poisson regression, to calculate prevalence ratios (RR) and $95 \%$ confidence intervals (95\%CI), considering the complex sampling used. Results: a total of 4.001 people were interviewed $-52.8 \%$ were women, $72.2 \%$ were brown and $19.7 \%$ were hypertensive -; the prevalence of self-reported chronic kidney disease was 2.1\% (95\%CI 1.6 to 2.5), positively associated with age (on years: $35-44, P R=2.31,95 \% C I$ 1.02 to $5.21 ; 45-59, P R=2.52,95 \% C I 1.10$ to $5.75 ; \geq 60$, $P R=2.95,95 \% C I 1.21$ to 7.16$)$ and stroke $(R P=2.20$, 95\% CI 1.09 to 4.45). Conclusion: two out of 100 manauaras reported kidney disease, more frequent in older adults and stroke.

Keywords: Renal Insufficiency, Chronic; Adult; Self-Report; Prevalence; Population; Cross-Sectional Studies. perspectives. Lancet [Internet]. 2013 Jul [cited 2020 Jan 10];382(9888):260-72. Available from: https:// www.thelancet.com/journals/lancet/article/PIIS01406736(13)60687-X/fulltext. doi: 10.1016/S01406736(13)60687-X

16. Stevens PE, Levin A. Evaluation and management of chronic kidney disease: synopsis of the kidney disease: improving global outcomes 2012 clinical practice guideline. Ann intern Med [Internet]. 2013 Jun [cited 2020 Jan 10];158(11):825-30. Available from: https://annals.org/aim/fullarticle/1691737/evaluationmanagement-chronic-kidney-disease-synopsis-kidneydisease-improving-global. doi: 10.7326/0003-4819158-11-201306040-00007

17. Levey AS, Eckardt KU, Tsukamoto Y, Levin A, Coresh $\mathrm{J}$, Rossert J, et al. Definition and classification of chronic kidney disease: a position statement from Kidney Disease: Improving Global Outcomes (KDIGO). Kidney int [Internet]. 2005 Jun [cited 2020 Jan 10];67(6):2089-100. Available from: https://www.kidney-international.org/article/S00852538(15)50698-4/fulltext. doi: 10.1111/j.15231755.2005.00365.x

\section{Resumen}

Objetivo: estimar la prevalencia de la enfermedad renal crónica autoinformada y factores asociados en adultos residentes en la Región Metropolitana de Manaus, Brasil. Métodos: estudio transversal de base poblacional realizado en 2015, con muestreo probabilístico para seleccionar adultos $\geq 18$ años; los participantes fueron entrevistados en domicilio; los factores asociados al autoinforme de enfermedad renal fueron investigados por la regresión de Poisson, para calcular las razones de prevalencia (RP) jerárquica e intervalos de confianza del 95\% (IC95\%), considerando el muestreo complejo utilizado. Resultados: fueron entrevistadas 4.001 personas - 52,8\% mujeres, $72,2 \%$ pardos y 19,7\% hipertensos -; la prevalencia de enfermedad renal autoinformada fue del 2,1\% (IC95\% 1,6;2,5), asociada positivamente con la edad (en años: 35-44, $P R=2,31$, IC95\% 1,02 a 5,21; 45-59, PR=2,52, IC95\% 1,10 a 5,75; $\geq 60$, $P R=2,95, I C 95 \%$ 1,21 a 7,10) y accidente cerebrovascular (PR=2,20, IC95\% 1,09 a 4,45). Conclusión: dos de cada 100 adultos informó enfermedad renal crónica, más frecuente en adultos mayores con accidente cerebrovascular.

Palabras clave: Insuficiencia Renal Crónica; Adulto; Autoinforme; Prevalencia; Población; Estudios Transversales.

Recebido em 02/04/2019

Aprovado em 23/10/2019 